\title{
EL CONSERVADURISMO EPISTÉMICO NO IMPLICA NECESARIAMENTE COHERENCIA
}

\section{CONSERVATISM DOES NOT NECESSARILY ENTAIL COHERENCE}

\author{
Rodrigo Laera ${ }^{1}$ \\ Universidad de Barcelona, CONICET
}

Recibido: 29/09/2016

Aceptado: 02/01/2017

Resumen: El objetivo de este trabajo es argumentar que no hay un requisito de coherencia necesario para sostener la racionalidad del conservadurismo epistémico. Así, se expondrán dos argumentos que cuestionan la idea de que la coherencia es uno de los motivos para mantener nuestras creencias justificadamente. El primer argumento se basa en la paradoja de la lotería y el segundo argumento se basa en la imposibilidad de recordar la razón o la evidencia por el cual uno cree que $p$. Luego se expondrá la diferencia entre coherencia diacrónica y sincrónica para sostener que ninguna de las dos están implicadas por la posición conservadora, ni aun como un desiderátum epistémico. Por último, se explica una de las causas por las que intuitivamente asumimos la necesidad de coherencia para mantener nuestras creencias en el tiempo.

Palabras claves: conservadurismo, coherencia, creencia, desiderátum epistémico.

\begin{abstract}
The aim of this paper is to argue that there is no requirement of coherence needed to sustain the rationality of epistemic conservatism. Thus, two arguments that challenge the assumption that coherence is one of the reasons to keep our beliefs justifiably will be presented. The first argument draws on the lottery paradox and the second argument builds upon the inability to remember the reason or evidence to which one believes that $p$. Then, the difference between diachronic and synchronic coherence will be expose to sustain that the coherence is neither entailed by the conservative position, not even as an epistemic desideratum. Finally, one of the causes why we intuitively assume the necessity of coherence to retain our beliefs in the time is explained.
\end{abstract}

Keywords: Conservatism, coherence, belief, epistemic desideratum.

1. (rodrigolaera@gmail.com) Rodrigo Laera es Investigador de la Universidad de Barcelona dentro del proyecto "El horizonte de lo común (entre una subjetividad no personal y una comunidad no identitaria) además de ser Profesor Asociado de Filosofía, Ética y Estética de la Universidad Argentina de la Empresa. 


\section{Introducción}

El conservadurismo epistémico sostiene que las creencias que poseen los sujetos no se cambian ni revisan sin tener alguna razón para ello. De modo que un sujeto $S$ está justificado en continuar aceptando $p$ en ausencia de una razón especial para aceptar no-p (Foley, 1983; Hartman 1986, 1999; Christensen, 1994; Sherman \&Harman, 2011; Laera, 2013). En varias ocasiones se ha sostenido que el conservadurismo epistémico requiere que la aceptación de $p$ lleva implícita su adecuación con un conjunto de creencias aceptadas como verdaderas y que excluyen no-p, asumiendo un principio de coherencia epistémica. Uno tiende a aceptar creencias que son coherentes con el acervo de creencias que se posee y rechazar las que no lo son. Por ejemplo, Chisholm (1984) sostuvo que la coherencia es un desiderátum epistémico para que las creencias sean aceptables. Por lo tanto, afirmar que para todo $S$, si $S$ acepta una proposición que no es explícitamente contradictoria por algún conjunto de proposiciones, entonces tal proposición tiene alguna presunción a su favor -0 es epistémicamente aceptable- para $S$. Más recientemente, pero en el mismo orden, también McCain (2008) sostuvo que un principio de coherencia es necesario para mantener justificada una creencia. De manera que si $S$ cree que $p$ y $p$ no es incoherente, entonces $S$ está justificado en retener la creencia que $p$ y $S$ permanece justificado en creer que $p$ tan lejos como $p$ no sea falseada por $S$.

El presente trabajo considera que el requisito de coherencia -incluso como fue presentando por Chisholm o McCain- no es una condición necesaria para mantener o abandonar creencias. Para demostrar esto se expondrán dos argumentos: uno basado en la paradoja de la lotería y otro basado en el problema que tienen los sujetos de recordar evidencias. El primer argumento sostiene, descriptivamente, que los individuos atraviesan circunstancias en las que dejan de lado la coherencia de sus creencias cuando toman decisiones racionales y, sobre todo, cuando las mantienen justificadamente. El segundo argumento afecta, evaluativamente, al desiderátum chishomiano de la necesidad de coherencia, al considerar que las creencias que implican una contradicción con un conjunto creencias ya aceptado como verdadero no deberían considerarse irracionales.

\section{Argumento basado en la paradoja de la lotería}

Supóngase que el grado de confianza en una creencia pueda ser medido en una escala probabilística que va de 0 a 1 , donde 1 representa 
la certeza del sujeto y 0 representa una completa incertidumbre. Ahora imagínese un concurso de lotería en el que participan 100 números y que solamente uno de los números será el ganador, de manera que no hay ninguna razón para pensar que un número tiene más posibilidades que otro. Así, sería racional creer que hay un 0.99 de confianza subjetiva que el número comprado no va a ganar y un 0.01 de que sí lo va a hacer. Por lo tanto, desde el punto de vista práctico, sería racional no comprar ningún billete ${ }^{2}$.

No obstante, si la decisión de comprar un billete solo se apoyara en las evidencias probabilísticas, entonces parece racional creer que, tomados uno por uno, ningún número ganará, aunque también sea racional creer que hay uno que va ser el ganador, aunque nadie sepa cuál es ${ }^{3}$. En definitiva, si se piensa en la coherencia de las creencias -y que excluye las conjunciones contradictorias entre evidencias-, entonces ninguna de las dos creencias parecería ser racional, ni se podría mantener justificadamente.

Ahora bien, ¿qué sugiere esta paradoja? Obviamente, cuando alguien compra un número de lotería piensa que tiene alguna probabilidad de ganar, aunque sea muy pequeña, pues siempre se puede tener un carácter racionalmente optimista. Pero también se puede ser racionalmente pesimista y creer que el número a comprar no va resultar ganador, pensando en la escasa probabilidad. La cuestión es que no hay nada irracional en mantener ambas opiniones en tiempos diferentes y ante objetivos pragmáticos distintos. Si $S$ sostiene la creencia de que el número puede y no puede resultar ganador, entonces la coherencia de dichas creencias no es un requisito para atribuirles racionalidad, ni para conservarla o abandonarla racionalmente. De hecho una misma persona puede seguir pensando que su número no va a ganar sobre la base del 0.99 de probabilidad y también puede ganar sobre la base de 0.01 de probabilidades -siempre hay un número que gana.

Desde el punto de vista conservador, $S$ seguiría manteniendo la primera actitud, aunque también conserve la segunda cuando se le reproche su excesivo optimismo. La ambigüedad de $S$ para mantener sus creencias se debe a que sus objetivos pragmáticos, que dan lugar a los criterios en los que se apoyan ambas creencia, son distintos. De tal manera que la

2. Foley (1992) presenta esta paradoja como una consecuencia de la tesis lockiana de los grados de creencias. Esta tesis sostiene que es epistémicamente racional creer en p solo si se tiene un grado de confianza que sea suficiente para la creencia en p. Así, se cree racionalmente que p aún si el específico grado de creencia es algo más alto o algo más bajo que lo que debería dar la evidencia.

3. Para una explicación alternativa de este problema, véase Hawthorne (2004).

Thémata. Revista de Filosofía №56 (2017) pp.: 199-212. 
contradicción en este caso no es motivo suficiente para cambiar o abandonar lo que estaría creyendo.

Si la contradicción no es suficiente como para que alguien cambie sus creencias ¿por qué o cómo, entonces, alguien habría de cambiarlas o abandonarlas racionalmente? ¿Cuál sería el criterio adecuado para que alguien cambie o abandone racionalmente sus creencias? La respuesta es que, prima facie, el cambio de creencias se debe a decisiones pragmáticas. De modo que la elección de opciones está influenciada por la acción de tomar una decisión; así, si $S$ cree que creer que $p$ es lo mejor puede hacer, entonces obrará en consecuencia y creerá que $p^{4}$. Dicha elección no tiene que ver con un corpus coherente de conocimiento en el cuál encaja la creencia para que se mantenga, ni en una comparación sincrónica de creencias competidoras. Por el contrario, tiene que ver con una cuestión de convicción respecto a la información disponible y de ser concluyente cuando se necesita serlo.

Piénsese, en lugar de la paradoja de la lotería, en el problema escéptico. Cuando uno duda que tiene dos manos teniendo en cuenta que puede ser un cerebro en una cubeta, lo que hace es elegir una creencia sobre su competidora de manera sincrónica. En contextos cotidianos, uno sabe o cree que tiene dos manos porque no se tienen en cuenta creencias competidoras. En cambio, en contextos más específicos, como el filosófico, se pone entre paréntesis las creencias cotidianas para evaluar los desafíos escépticos. Todo esto sin importar que ambas creencias son excluyentes o contradictorias. Uno puede mantener ambas creencias en distintas circunstancias. Cambiando el contexto, cambiamos también la forma en la que nos manejamos, nuestras actitudes respecto al mundo y, por supuesto, nuestras creencias. Podemos ser concluyentes de manera contradictoria $\sin$ que esto afecte nuestra racionalidad ${ }^{5}$.

\section{El argumento basado en el problema recordar evidencias}

La exigencia de que las creencias actuales deben cohesionar con las creencias que uno tiene implícitamente o que espera tener, parece responder a cierta intuición de que la coherencia de las creencias en el tiempo es un desiderátum epistémico. En este sentido, se debe diferenciar la prag-

4. La noción de "lo mejor" es controvertida si se la toma de manera constitutiva, menos problemático es tomarla de manera regulativa como "lo más conveniente" o "lo más útil" o "lo más cercano a la verdad" o cualquier virtud epistémica que funcione como un atributo que haga deseable la creencia.

5. Para otra explicación partiendo de la paradoja del prefacio, véase Kvanvig (2012).

Thémata. Revista de Filosofía $\mathrm{N}^{\circ} 56$ (2017) pp.: 199-212. 
mática de las atribuciones de conocimiento de la epistemología propia del desiderátum. La primera se da a partir de los usos de las expresiones del tipo " $S$ sabe que $p$ ", el segundo se refiere a las reglas constitutivas de lo que debería suceder para que $S$ sepa que $p$. En cualquier caso, la coherencia no es un factor decisivo cuando se trata de conservar nuestras creencias.

Para determinar satisfactoriamente que dos proposiciones sean coherentes resulta imprescindible que uno evalúe las implicaciones de ambas. Justamente, el conservadurismo brinda la posibilidad de mantener la racionalidad de nuestras creencias aun si olvidamos las razones por las que han surgido. Esto se conoce como el fenómeno de las evidencias perdidas. Dicho de otra manera, el fenómeno de la evidencia perdida es aquel en que los sujetos que inicialmente fundamentan sus creencias sobre evidencias adecuadas que las justifican, pero subsecuentemente olvidan en qué consistían tales evidencias - de la misma manera que olvidan el preciso momento en el que cambian de creencias. Así, $S$ puede creer racionalmente $p$ aunque las evidencias se encuentren perdidas en el tiempo. Si esto no fuera así -si siempre necesitáramos recordar evidencia con la que apoyar nuestras creencias- nos encontraríamos ante el excesivo intelectualismo que significa tener que contrastar las creencias que se vienen dando con un cúmulo de creencias aceptadas con anterioridad que funcionarían como evidencias. La mayoría de las veces uno no recuerda por qué cree lo que cree. En consecuencia, el fenómeno de las "evidencias perdidas" resulta imprescindible para tratar con el mundo.

Si la esencia del conservadurismo radica en la idea de que los sujetos revisan sus creencias cuando son inducidos a ello, entonces el fenómeno de la evidencia perdida conduce tanto a una ventaja como a una desventaja. La ventaja yace en que concuerda con el principio de economía propio de la vida diaria, dando así respuesta al escepticismo: somos incapaces de indagar sobre las fuentes de todas nuestras creencias para justificarlas. La desventaja radica en que se puede continuar creyendo que $p$ es verdadero cuando es falso, y mantener esa creencia intocable a lo largo del tiempo. A esto se le sumaría que uno puede justificar cierta desidia epistémica enmascarándola con una posición conservadora; en efecto, si $S$ puede seguir creyendo $p$ sin que nada motive el cambio de creencia, $S$ no parece epistémicamente obligado a buscar evidencias que fundamenten su postura. Ambas, ventaja y desventaja, convergen en el hecho de que uno no pueda recordar la evidencia que originalmente impulsó una creencia, hace que sea poco atractivo requerir que uno recuerde muchas otras de nuestras evidencias originales para que encajen o sean coherentes con una creencia actual.

Más allá de que en ocasiones resulte complicado identificar las fuentes de nuestras creencias, las creencias también cambian sin que 
nos demos cuenta de ello. Que muchas creencias cambien sin motivo aparente presenta un nuevo desafío para el conservadurismo. Por supuesto, siempre es posible buscar y encontrar motivos por los que racionalizar creencias y, muchas veces, los resultados de esta búsqueda están guiados por los intereses y las circunstancias de quienes buscan atribuir motivos. Este tipo de racionalización conforma la teleología de la razón práctica; al justificar por qué se cree lo que se cree, los sujetos se desenvuelven en el plano de la decisión y de la acción conforme a fines -por ejemplo, el acto de aceptar que $p$ conduce a la verdad conlleva ventajas prácticas si es que $p$ es verdadera. Sin embargo, el conservadurismo no se define recurriendo a las causas que llevaron a $S$ a optar por $p$, sino por el hecho de que alguna vez se optó por $p$, porque la creencia pareció, prima facie, más razonable que otras competidoras si las hubiere. Que muchas veces uno cambie de creencias sin que se dé cuenta de ello, implica una desventaja intuitiva que intenta subsanarse con la construcción de una narración que ilustre por qué motivo se produjo el cambio y que, por lo tanto, justifique la creencia actual aduciendo coherencia.

\section{La diferencia entre coherencia diacrónica y sincrónica}

Quizás tanto el argumento de la lotería como el de recordar evidencias en contra de la necesidad de coherencia en el conservadurismo epistémico se resuelva, en primer lugar, al distinguir satisfactoriamente entre dos tipos de coherencia: la sincrónica y la diacrónica. Para, en segundo lugar, establecer cierta disposición pragmática al momento de elegir ser coherente o al presentar la coherencia como un desiderátum epistémico. Esta es quizás la estrategia más plausible para apostar por la coherencia epistémica.

Siguiendo esta estrategia, se define la coherencia sincrónica (CS) de la siguiente manera:

CS: una creencia es sincrónicamente coherente cuando (i) está siendo sostenida sobre el equilibrio de un cuerpo total de creencias; o bien (ii) no está en conflictos con el cuerpo total de creencias.

Y la coherencia diacrónica (CD) de la manera siguiente:

$C D$ : una creencia es diacrónicamente coherente cuando (i) está sostenida sobre el equilibrio de un cuerpo total de creencias alguna vez asumidas como verdaderas; o bien (ii) no entra en conflicto con el equilibrio del cuerpo total de creencias que alguna vez fueron asumidas como verdaderas.

Dos aclaraciones respecto a las definiciones presentada. La primera es que la diferencia entre (i) y (ii) de CS y CD es la que hay entre la 
coherencia positiva y la negativa, una diferencia que para el propósito de este trabajo no es preciso por el momento resaltar. La segunda consiste en que CS y, sobre todo, CD no implican consistencia; es decir, que el conjunto de creencias haga lógicamente posible que cada miembro sea verdadero.

Con el caso de la lotería se criticó el uso sincrónico de la coherencia para conservar nuestras creencias racionalmente, en cambio con el caso de la evidencia perdida se criticó el uso diacrónico de la coherencia como desiderátum epistémico para conservar creencias racionalmente. Partiendo de esta diferencia se sostiene que en determinados contextos las creencias cambian debido a contradicciones sincrónicas con otras creencias, mientras que las contradicciones diacrónicas pueden ser aceptadas, a pesar de que no sea un desiderátum epistémico. Si la coherencia diacrónica no es necesaria para la conservación de creencias, entonces la razón para aceptar no-p no implica abandonar $p$. Por lo tanto, las creencias cambiarían arbitrariamente.

Llegados a este punto se hace necesario introducir una distinción entre un cambio de creencias y el abandono de creencias para preservar la coherencia sincrónica y diacrónica dentro del conservadurismo. La creencia de que $p$ puede cambiar a no- $p$, sin que no- $p$ sea abandonada, sino que se mantiene en el tiempo tácitamente. También a la inversa, $S$ podría abandonar explícitamente $p$, sin necesariamente creer en no-p explícitamente. En suma:

Abandono: S abandona p si y solo si encuentra una creencia verdadera incompatible con $\mathrm{p}$.

Cambio: S cambia de creencia p, si y solo si hay otra creencia q más adecuada a los objetivos epistémicos de $\mathrm{S}$.

Uno podría pensar que ambas -"abandono" y "cambio"- funcionan si se distingue entre coherencia diacrónica y sincrónica. Teniendo en cuenta esta diferencia, se puede relaborar el conservadurismo sosteniendo que, aunque $S$ mantenga creencias que son más adecuadas a los objetivos epistémicos, esto no quiere decir que uno abandone una creencia porque entra en conflicto otra ya aceptada previamente. Dicho de otra manera, que la coherencia sea una prioridad epistémica -si se presenta una creencia que entra en conflicto con otra ya aceptada, debe abandonarse- no implica que las creencias se correspondan unas con otras diacrónicamente. La cuestión es no caer en la tentación de que coherencia implica conjunción de creencias, de manera que $p$ y $q$ deben ser ambas verdaderas si el conjunto que encierra dicha conjunción sea coherente. Se volverá a esto más adelante.

Así, se debe rechazar $p$ si acepta no-p de manera sincrónica; es decir, cuando ambas creencias conforman estados doxásticos simultáneos. En cambio, si las creencias no fueran simultáneas, el requisito de compa-

Thémata. Revista de Filosofía №56 (2017) pp.: 199-212. 
rar creencias como en un acto de conjunción no sería necesario para CD -la conjunción sería meramente tácita, pues que una creencia cambie no significa que otras implicadas se abandonen. De esta manera se resolvería el problema de la lotería. Desde CD uno puede tener la creencia de que no va a ganar y cambiar esa creencia sin abandonarla, como cuando compra un billete pensando en que tiene alguna chance de hacerse millonario.

La misma estrategia puede ser utilizada para dar con una explicación del problema de recordar evidencias que incluya coherencia. Cuando alguien olvida una evidencia que soporta una creencia, permite que otra evidencia contradictoria, que también haya sido olvidada, soporte una creencia compatible con la anterior. Por ejemplo, uno puede tener la evidencia de que hay una cebra dentro de la jaula para creer que hay un animal dentro de la jaula y también puede tener la evidencia de que no hay una cebra dentro de la jaula, sino una mula ingeniosamente pintada para creer que hay un animal dentro de la misma. $S$ puede olvidar una de las dos evidencias, que la otra le es suficiente para sostener que hay un animal dentro de la jaula.

A lo dicho debe agregársele el problema de la sub-determinación de las creencias: ninguna evidencia disponible por sí sola resulta suficiente para determinar una creencia. Ampliando los ejemplos anteriores, supóngase que $S$ cree que Julio Cesar dijo "Jacta alea est" cuando cruzó el Rubicón sobre la evidencia del testimonio de Suetonio. Pero olvidando esto, $S$ también cree que los testimonios de Suetonio son exagerados al narrar el cruce del Rubicón sobre la evidencia de lo poco probable que resulta que alguien haya conocido la psicología de Julio Cesar. El olvido de la desconfianza sobre el testimonio de Suetonio, permite tener una creencia; mientras que también permite tener una creencia incompatible con ese testimonio, cambiando una por otra según la ocasión o el contexto. Solo cuando se arroja luz sobre las incompatibilidades, $S$ es capaz de decidir qué aceptar como creencia y cuál abandonar.

Esto también mostraría que el aporte coherentista parece ser solo teorético; cuando la mayoría de las veces no somos capaces de confrontar y comparar creencias opuestas junto con sus respectivas evidencias ${ }^{6}$. También muestra que la coherencia diacrónica no es un requisito epistémico -ni siquiera un desiderátum- para tratar con el mundo en nuestra vida diaria. $S$ conservaría sus creencias y sus evidencias sin pérdida de racionalidad; incluso sin ser epistémicamente irresponsable, dependiendo las circunstancias en las que se encuentre. En suma, las personas son capaces de sacar conclusiones muy diferentes de la misma evidencia, aunque no cometan errores en sus apreciaciones. De la misma manera que son capaces

6. Contra esto, véase Lycan (1998).

Thémata. Revista de Filosofía $\mathrm{N}^{\circ} 56$ (2017) pp.: 199-212. 
de tener una creencia $p$ soportada por una evidencia olvidada, para luego rechazar explícitamente la evidencia sin que con ello se rechace $p$.

Ahora bien, el postulado de coherencia para el conservadurismo puede modificarse en otro más débil, tanto sincrónicamente como diacrónicamente. En el siguiente sentido,

Versión débil de sincronía: (i) es racional sostener una creencia $p$, si no es sincrónicamente contradicha por otras proposiciones que tienen alguna presunción a favor (ii) es racional mantener una creencia $p$, si no es sincrónicamente contradicha con otras proposiciones aceptadas por la comunidad a la que pertenece $S$.

La pretensión de diacronía puede ser contrapuesta a la siguiente restricción:

Versión débil de diacronía: (i*) no es posible que $S$ crea y no crea que $p$ al mismo tiempo; (ii*) la creencia puede ser irracional, aunque no haya nada que la contradiga sincrónicamente, pero no puede ser racional cuando hay evidencias que la contradigan sincrónicamente.

Con respecto a (i) de la versión débil de sincronía, parece muy complicado aceptar que $S$ crea y no crea algo explícitamente al mismo tiempo. Una creencia dada deja su lugar a otra creencia y luego ambas pueden compararse. Sin embargo, el acto de comparación de creencias no requiere que ambas estén sucediendo al mismo tiempo. Quizás (i) se pueda interpretar de la manera en que el mismo acto de comparar conduzca a evaluar dos creencias como contradictorias. Y si esto sucede, $S$ debería abandonar aquella creencia que no tiene ninguna presunción a favor. No obstante, esta interpretación no dice nada acerca de la racionalidad de las justificaciones que puede aportar $S$ para mantener su creencia, pues $S$ puede tener una presunción a favor de $p$ basada simplemente en el deseo de tenerla, sobre todo cuando las evidencias no vienen a la mente de forma cartesiana, es decir, de manera clara y distinta ${ }^{7}$. Dicho de otro modo ¿cómo saber que la fuente de presunción de una creencia no es simplemente la evidencia, sino la evidencia que deseamos presentar en detrimento de otras que la contrarían? ¿Cómo saber que uno no se auto-engaña cuando presenta una evidencia o una justificación pensando que es la mejor disponible? Estas cuestiones son especialmente acuciantes, sobre todo si se considera que muchas de nuestras justificaciones son excusas, pues vienen después de las creencias solo para confirmarlas.

Con (ii) estas cuestiones parecen disiparse, ya que la comparación no es solo introspectiva, empezando y terminando en la mente del sujeto.

7. Si se considera que las evidencias aparecen de manera clara y distinta, entonces se podría conducir el análisis epistemológico hacia la idea de que las evidencias se caracterizan por ser irresistibles.

Thémata. Revista de Filosofía N56 (2017) pp.: 199-212. 
Para evitar esta suerte de mentalismo, se presenta un estándar social con el que comparar y regular las creencias. Pero aquí hay dos peligros: el relativismo y la imposibilidad de innovación. Con respecto al relativismo se debe señalar que, según (ii), las proposiciones aceptadas por la comunidad determinan si uno debe abandonar una creencia, como las proposiciones aceptadas de una comunidad a otra pueden variar entonces los criterios por los cuales uno debe mantener una creencia también. Esto hace que no haya criterios universales. En una comunidad existirá un criterio o una serie de proposiciones que permitan mantener una creencia, mientras que en otra comunidad puede haber otra serie de proposiciones que permitan mantener una creencia bien distinta. Es más, atribuir valor de verdad a una creencia bajo las normas de la razón o racionalidad, implica la circulación de muchos factores como la adquisición de información, la inferencia o la finalidad práctica. Si estos factores cambian de comunidad el criterio de racionalidad con el que evaluamos creencias también cambia. Y todo esto nos arrojaría en las aguas del relativismo. Con respecto a la imposibilidad de innovación, cabe especular que si se diera a rajatabla (ii), entonces parece imposible que uno pueda modificar el statu quo. Piénsese que, de mantener (ii), los grandes personajes de comienzos de la modernidad deberían haber mantenido creencias medievales, abandonando su "nueva" cosmovisión.

Igual de problemática es la versión débil de diacronía. Aunque alguien esté de acuerdo con $\left(\mathrm{i}^{*}\right)$, incluso con la primera parte de (ii*), tiene que aceptar que se pueda creer algo completamente alocado, aún si no encuentra creencias que lo contradiga. Por ejemplo, Juan sostiene que María es infiel, simplemente porque tiene ataques celos, sin que María presente evidencias acerca de su fidelidad pero su creencia puede ser perfectamente coherente -o no-contradictoria- con otras creencias como que María tiene muchos amigos. No obstante, $\left(i^{*}\right)$ y $\left(i i^{*}\right)$ ponen el foco en la racionalidad. Esto es que existen normas que rigen a los estados doxásticos y que están asociadas a las prácticas cognitivas que permiten tratar con el mundo. ¿Son estas prácticas siempre coherentes? Si no lo son ¿deberían serlo? Estas preguntas están implícitas en la segunda parte de (ii*). Sin embargo, ambas preguntas parecen no tener sentido o tener un sentido metafórico. Las acciones se ejecutan, pueden ser una causa o una consecuencia de otra acción y también pueden poseer motivos racionales para su ejecución, pero no pueden atribuírseles coherencia. Las acciones no son coherentes, sino los motivos que conducen a su ejecución. Las personas solo tratan con el mundo, aunque este trato se produzca de acuerdo a intereses, deseos, necesidades o motivos racionales.

Como se ha dicho, lo que sí puede ser coherente es la racionalidad que depositamos en las acciones. De modo que tener una razón o un motivo 
para ejecutar una acción es algo bien distinto al trato con el mundo bajo la lupa de la coherencia. Es cierto que la razón posee un aspecto teleológico, que se entiende mediante de la reflexión sobre la práctica racional. No obstante, uno no es irracional por tratar con el mundo sin pensar o reflexionar o tener creencias acerca de lo que hace. Muchas de nuestras prácticas son indeliberadas, mecánicas, y no por eso son irracionales.

Aunque la razón posea la función elemental de orientarnos hacia la verdad, pues la exposición y la deliberación acerca de la verdad generalmente ocurren al margen de los intereses individuales, la atribución de verdad puede pasar a un segundo plano o puede ser manipulada a pesar de que haya evidencias que la contradigan. Así, (ii*) omite el fenómeno de la evidencia perdida, pues ¿cómo saber que hay evidencias que contradicen una creencia si en la mayoría de los casos, cuando evaluamos nuestras creencias, no las contrastamos conscientemente con evidencias, a veces por descuido, a veces por olvido y, muchas otras, por la dificultad intelectual que envuelve al acto de comparar? En este sentido, sostener cualquier tipo de coherencia como una condición necesaria para mantener o abandonar creencias conduce a una posición extremadamente intelectualista respecto a la racionalidad y a nuestra manera de tratar con el mundo.

\section{5. ¿Por qué parece intuitivo que las creencias se manten- gan bajo la condición de ser coherentes?}

Se ha sugerido que la coherencia -tanto diacrónica como sincrónica, en sentido fuerte o débil- no hace que mantengamos o abandonemos nuestras creencias, no al menos como un factor determinante o una condición necesaria. Sin embargo, ¿por qué se produce esa suerte de exigencia cuando evaluamos la racionalidad desde una perspectiva teorética? Dicho de otra manera, ¿cuál es el motivo por el que la coherencia parece ser una exigencia decisiva cuando pretendemos conservar nuestra racionalidad?

El principal presupuesto que responde a la anterior pregunta consiste en intentar sostener un principio de conjunción epistémica, análogo al de la lógica, como fuente de racionalidad. Si una evidencia justificara $p$ y otra o la misma evidencia justificara $q$, entonces la conjunción $p$ y $q$ estría justificada.

El principio de conjunción epistémica es una intuición clave de nuestra pretensión de racionalidad, pues parece irracional justificar $p \mathrm{y}$ luego justificar $q$, para después admitir que la conjunción $p$ y $q$ no se encuentran justificadas. El caso de la lotería expuesto anteriormente siembra dudas acerca de dicha intuición. $S$ puede justificar la compra de un 
billete de lotería apoyado en la evidencia de que todos billetes tienen la misma probabilidad de ganar y afirmar justificadamente que el billete que ha comprado no ganará. Sin embargo, $S$ no puede justificar conjuntamente la compra del billete y la creencia de que no va a ganar. Un caso similar: $S$ puede justificar sobre evidencia disponible la creencia que es necesario un seguro de vuelo y también, separadamente, la creencia de que deber hacer la reservación en el hotel de destino. Sin embargo, la conjunción de ambas creencias parecen irracionales, pues si $S$ sabe que va a llegar a destino ¿por qué sacaría un seguro de vuelo? ${ }^{8}$

Además, aunque resulte obvio, cabe señalar que no cualquier conjunción implica coherencia. Por ejemplo, $S$ puede creer que la hierba es verde y que las mariposas vuelan, siendo ambas proposiciones verdaderas, sin que haya una relación coherente entre ambas creencias. Para evitar esto, se suele considerar que la coherencia exige dos procesos: el de comparar y el de inferir. Sin embargo, ambos procesos -o capacidades- dejan de lado dos virtudes fundamentales del conservadurismo. La primera virtud radica en la acumulación de conocimiento; la segunda virtud en la economía de la reflexión.

Respecto a la primera virtud, mantener creencias sin necesidad de revisarlas permite el avance en la cadena de creencias, de manera que se acumule conocimiento. Si fuera necesario comparar o inferir creencias como propone el requisito de coherencia, entonces se haría difícil la acumulación, pues el acto de comparar -como el de inferir- necesita tiempo para la correspondiente revisión del conocimiento alcanzado. Respecto a la segunda virtud, el trabajo epistémico sería demasiado arduo, aun cuando se tratase de conocer las cosas más simples de la vida. Esto viola el principio de economía epistémica, este principio nos advierte que uno no debe empeñar su tiempo -que es muy limitado- en creencias o cuestiones intrascendentes. Dicho de otra manera, dado que nuestras ocupaciones ocupan gran parte del tiempo, no debemos dedicarnos a comparar o inferir creencias cuando el asunto en cuestión no es considerado importante.

Ambas virtudes -acumulación y economía- son, justamente, una parte significativa de lo que hace deseable y plausible al conservadurismo epistémico. Si el conservadurismo pierde estas dos virtudes, entonces sería una teoría poco atractiva, pues perdería con ellas su carácter descriptivo. En este sentido, el carácter descriptivo es también un desiderátum epistémico; lo que sucede cuando alguien conserva sus creencias justificadamente sin una revisión ni una comparación es también lo más deseable. Que el

8. La misma objeción puede extenderse a la noción de clausura, donde para cualquier tema $T$, y cualquier proposición $p$, $q$, si $S$ sabe $p$ y $q$, entonces sabe $T$ (p \& q).

Thémata. Revista de Filosofía №56 (2017) pp.: 199-212. 
carácter normativo y el carácter descriptivo coincidan para la conservación de las creencias, también quiere decir que si alguien no tiene una razón o una justificación para conservar su creencia no por ello se vuelve una persona irracional. Este es un gran mérito del conservadurismo como teoría. Es más, no resultaría necesario demandar coherencia, pues si la coherencia deja de ser un requisito descriptivo, pasaría a ser simplemente normativo y con ello, ambos caracteres se separan, dejando al conservadurismo sin razón de ser. Justamente, el conservadurismo es una teoría plausible porque el carácter normativo y el descriptivo de cómo mantenemos nuestras creencias coincide.

\section{Conclusión}

Si el conservadurismo es verdadero, entonces que olvidemos las evidencias es un fenómeno que no implica necesariamente irracionalidad. Si olvidar evidencias no implica necesariamente irracionalidad, entonces que no se encuentre coherencia en las evidencias tampoco implicará necesariamente irracionalidad. En virtud de sus ventajas pragmáticas, el conservadurismo parece necesario para tratar con el mundo, si la coherencia es también necesaria entonces el conservadurismo no tiene razón de ser como teoría. No obstante, el coherentismo posee ventajas teóricas, pues puede transformarse no solo en un criterio de decisión, sino también en un criterio de aceptación. La cuestión es por cuál teoría decidirse

\section{Bibliografía}

Chisholm, R. (1982). The Foundations of Knowing. Minneapolis: University of Minnesota Press.

Christensen, D. (1994). Conservatism in Epistemology. Nous, 28, 69-89.

Foley, R. (1983). Epistemic Conservationism. Philosophical Studies, 165-182.

Foley, R. (1992). The Epistemology of Belief and the Epistemology of Degrees of Belief. American Philosophical Quarterly, 29(2), 111-124.

Harman, G. (1986). Change in View: Principles of Reasoning. Cambridge: MIT Press.

Harman, G. (1999). Reasoning, Meaning, and Mind. Oxford: Oxford University Press.

Hawthorne, J. (2004). Knowledge and Lotteries. Oxford: Clarendon Press.

Kvanvig, J. (2012). Coherentism and justified inconsistent beliefs: 
A solution. The Southern Journal of Philosophy, 50(1), 21-41.

Laera, R. (2011). Los desvios de la razón: el lugar de la facticidad en la cadena de justificaciones. Buenos Aires: Miño y Dávila.

Lycan, W. G. (1988). Judgement and Justification. Cambridge: Cambridge University Press.

McCain, K. (2008). The Virtues of Epistemic Conservatism. Synthese, 164, 185-200.

Sherman, B., \& Harman, G. (2011). Knowledge and assumptions. Philosophical Studies, 156, 131-140. 